\title{
Characterization of Polycaprolactone Films Biodeterioration by Scanning Electron Microscopy
}

\author{
Kamila Hrubanova $^{1,2}$, Stanislava Voberkova $^{3}$, Sona Hermanova ${ }^{4}$ and Vladislav Krzyzanek ${ }^{1}$ \\ ${ }^{1}$ Institute of Scientific Instrument ASCR, Department of Electron Microscopy, Brno, Czech Republic. \\ 2. Brno University of Technology, Institute of Physical Engineering, Brno, Czech Republic. \\ ${ }^{3}$ Mendel University in Brno, Institute of Chemistry and Biochemistry, Brno, Czech Republic. \\ 4. Institute of Chemical Technology Prague, Department of Polymers, Prague, Czech Republic.
}

Widespread studies on the biodegradation of plastics have been carried out in order to overcome the environmental problems associated with synthetic plastic waste. From this point of view aliphatic polyesters represent a class of biodegradable materials with potential for various applications. Research interest has been also paid on enhancement of biodegradation of resistant plastics by incorporation of aliphatic ester linkages to aromatic ones or modification of polyolefins with aliphatic polyesters [1]. Polycaprolactone (PCL) as representative of aliphatic polyesters undergoes biodegradation by the action of enzymes and microorganisms and hence it is suitable candidate for biodeterioration and biodegradation study.

Biodeterioration activity of Bacillus strain, naturally occurring in soil environment, on the surface of polycaprolactone films in mineral salt and mineral salt-yeast extract enriched media at $30{ }^{\circ} \mathrm{C}$ after 21 days was studied. Polyester square specimens $(10 \times 10 \mathrm{~mm})$ were sectioned from film prepared by melt pressing. Control abiotic tests were carried out by immersion of studied samples into the media in the absence of microorganism. Changes in film surface were evaluated by means of scanning electron microscopy (SEM) using Magellan 400/L (FEI, Eindhoven) at primary energy of $2 \mathrm{keV}$. For characterization of the material surface, here presented samples were without any chemical treatment and air-dried; they were coated by $4 \mathrm{~nm}$ of Platinum.

During 42 days of solid state cultivation of microorganism only unevenly distributed microcracks were observed on the polyester film surface. During submerged cultivation considerable acceleration of film surface deterioration was observed already after 21 days of test. The characterization of samples after the exposure to microorganism revealed synergic occurrence of enzyme-catalyzed and abiotic hydrolytic degradation. The abiotic hydrolysis was base-catalyzed since $\mathrm{pH}$ values in the range of 8-10 were developed in media during microorganism cultivation as the consequence of metabolic pathway. Microorganism and namely enzymatic action proceeds on ester bonds close to chains end since decrease in number-average molecular weight $\left(\mathrm{M}_{\mathrm{n}}\right)$ of polyester was measured. The process initiated with chain scission of ester bonds on the film surface was followed by loss of material (up to $3 \mathrm{wt} . \%$ ) as surface erosion. Surface erosion pattern developed is ascribed to the formation of degradation products ended with carboxylic groups in dissociated forms as the first step. Subsequently, the accumulation of more hydrophilic species supports both acceleration of chain degradation through autocatalysis and diffusion of water.

For the characterization of the investigated material surface structure by SEM, the parameters were evaluated [2]. There are following deformations recognized and described in the study: 1. Microcracks on the surface with sharp edges (long $0.1-5 \mu \mathrm{m}, 0.5 \mu \mathrm{m}$ in width, the depth of cracks up to a few $\mu \mathrm{m}$ ) and their directional orientation; 2. Holes in the surface (often annular); 3. The lamellar structure in the 
surface (incidence of long fibers with a diameter of up to $0.1 \mu \mathrm{m}$ and their clusters); 4 . The surface roughness; 5 . Places with smooth surfaces; and 6. The occurrence of bacteria (biofilm). The flat surface of PCL films is presented at Figure 1a. Microcracks developed on the surface of biotically aged films in mineral salt and mineral salt with yeast extract media were randomly distributed (Figure 1b, 1d). Formation of the irregular holes on the surface may be associated with higher enzymatic activity in mineral salt medium enriched with yeast extract. Polycaprolactone sample due to semicrystalline and hydrophobic character displayed only low water uptake in abiotic control media and consequently no weight loss along with no surface erosion proceeded (Figure 1c, 1e).

\section{References:}

[1] B Nowak, J Pajak, M Drozd-Bratkowicz and G Rymarz, Int. Biodeterior. Biodegrad. 65 (2011), p. 757.

[2] G Coulon, G Casteleina and C G'Sellb, Polymer 40 (1999), p. 95.

[3] VK and KH acknowledge financial support from CZ.1.07/2.3.00/20.0103 and LO1212 (EC and MEYS CR).

[4] SH thanks Ministry of Education, Youth and Sports of the Czech Republic (Grant MSM no.6046137302) for financial support
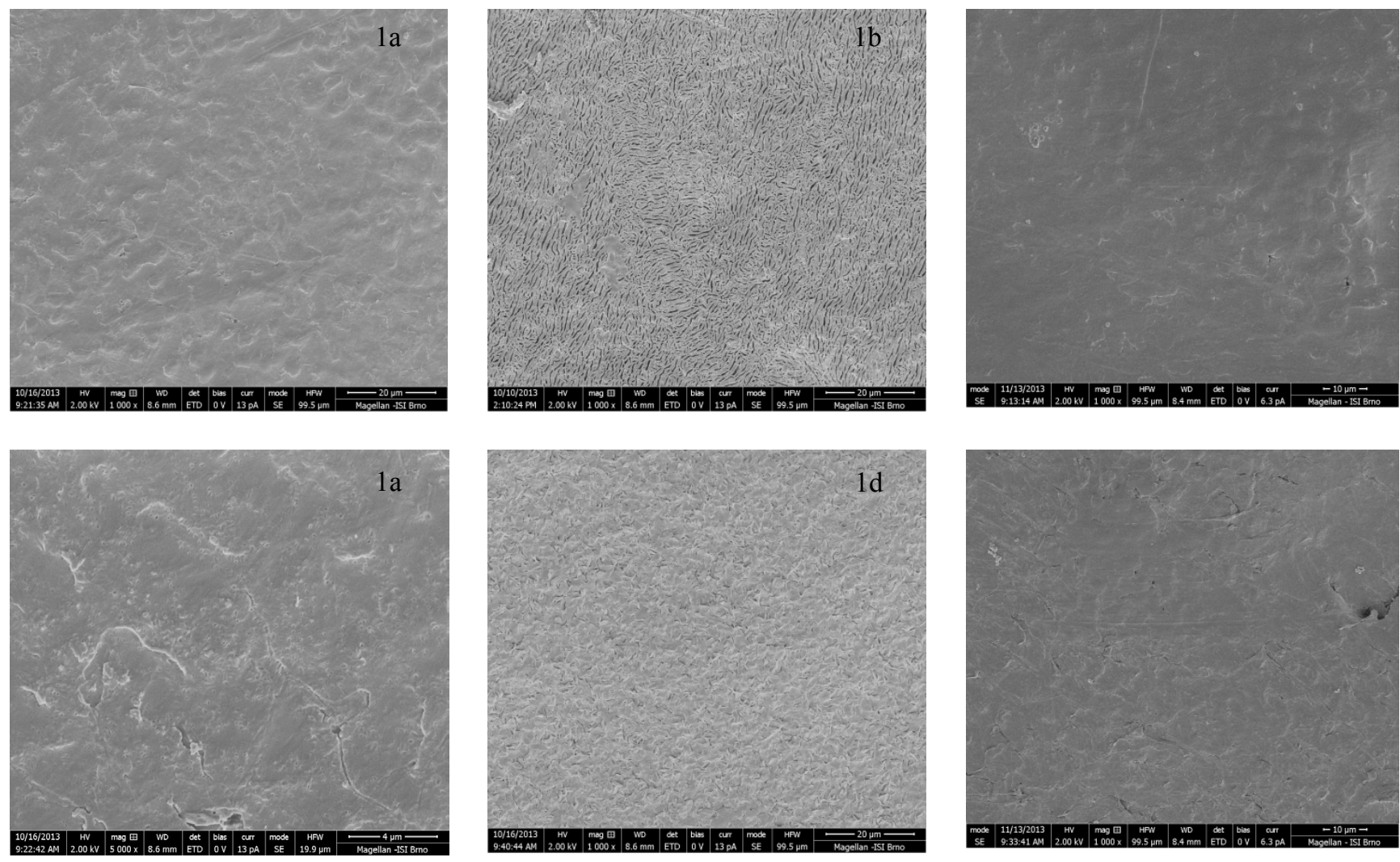

Figure 1. Scanning electron micrographs of polycaprolactone films. (1a) the original sample with higher magnification at bottom, (1b) PCL sample after 21 days in mineral salt medium inoculated with Bacillus strain, (1c) the control sample in mineral salt medium, (1d) PCL sample after 21 days in mineral salt medium supplemented with yeast extract and inoculated with Bacillus strain, (1e) the control sample in mineral salt medium supplemented with yeast extract. 